Pacific Journal of Mathematic 


\section{THE SUSPENSION OF THE GENERALIZED PONTRJAGIN COHOMOLOGY OPERATIONS}

\section{EMERY THOMAS}

1. The main theorem. In a previous paper [9] I have defined a sequence of new cohomology operations, called the generalized Pontrjagin operations. These operations use as coefficient groups the summands of a certain type of graded ring: namely, a ring with divided powers (defined by H. Cartan in [1]), which is termed a $\Gamma$-ring in [9]. Let $A=\sum_{k} A_{k}$ be a ring with divided powers such that each summand $A_{k}$ is a cyclic group of infinite or prime power order; we termed such rings p-cyclic in [9]. Then, the Pontrjagin operations are functions

$$
\mathfrak{F}_{t}: H^{2 n}\left(X ; A_{2 k}\right) \longrightarrow H^{2 t n}\left(X ; A_{2 t k}\right) \quad(k, n>0 ; t=0,1, \cdots)
$$

where $H^{q}(Y, B ; G)$ denotes the $q$ th (singular) cohomology group of the pair $(Y, B)$ with coefficients in the group $G$.

Let $C$ be a cohomology operation relative to integers $r, s$ and coefficient groups $G, I$. That is, $C$ is a natural transformation

$$
C: H^{r}(Y, B ; G) \longrightarrow H^{s}(Y, B ; \Pi) \text {. }
$$

With each operation $C$ we associate a second operation, $S(C)$, called the suspension of $C . S(C)$ is a natural transformation

$$
H^{r-1}(Y, B ; G) \longrightarrow H^{s-1}(Y, B ; I I) ;
$$

its definition is given in $\S 3$.

The purpose of this note is to determine $S\left(\mathfrak{F}_{t}\right)$, where $\mathfrak{F}_{t}$ is the generalized Pontrjagin operation. In order to state our result concerning $S\left(\mathfrak{P}_{t}\right)$, we need an additional cohomology operation, the Postnikov square (see [3], [10]). This was defined in [9], but only for a restricted class of coefficient groups. In this paper we will define the Postnikov square as a cohomology operation

$$
\mathfrak{p}: H^{q}\left(Y, B ; A_{2 k}\right) \longrightarrow H^{2 q+1}\left(Y, B ; A_{4 k}\right), \quad(q, k>0)
$$

where $A_{2 k}$ is an even summand of a $p$-cyclic ring with divided powers.

We now may state the main result of the paper.

THeorem I. For any cohomology operation $C$, let $S(C)$ denote the suspension of the operation $C$. Then,

Received October 18, 1957, in revised form December 19, 1958. This research has been partly supported by U. S. Air Force contract AF 49 (638)-79. 


$$
\begin{aligned}
& S\left(\mathfrak{F}_{2}\right)=\mathfrak{p} \\
& S\left(\mathfrak{F}_{t}\right)=0,
\end{aligned}
$$

where 0 denotes the zero cohomology operation.

The proof of Theorem I is given in $\S 5$. In $\S 2$ we define the operation $\mathfrak{p}$, while in $\S 3$ we give the definition of the suspension. In $\S 4$ we discuss relative cohomology operations, while in $\S 6$ we give some addi-

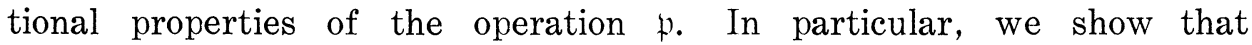
$S(\mathfrak{p})=0$. Finally, the last section gives the theorem, $\delta S(C)=C \delta$, for any operation $C$.

I would like to thank Professor N. E. Steenrod for the valuable suggestions made to me at the time of revising the paper. In particular the definition of the suspension in $\S 3$ and Theorem 7.1 are due to him.

2. The definition of the Postnikov square. The definition of the Postnikov square, $\mathfrak{p}$, is obtained by first defining a "model operation", $p$, which uses only a restricted category $\mathscr{C}$ of coefficient groups. The category $\mathscr{C}$ is defined as follows: let $Z_{r}=Z \mid r Z(r=0,1, \cdots)$, where $Z=$ integers $=Z_{0}$. Denote by $\mathscr{C}$ the category of all groups of the form $Z_{\theta}$, where $\theta$ is zero or a power of a prime. For each group $Z_{\theta}$ in $\mathscr{C}$ we have defined a $p$-cyclic ring with divided powers,

$$
G\left(Z_{\theta}\right)=G_{0}\left(Z_{\theta}\right)+\cdots+G_{t}\left(Z_{\theta}\right)+\cdots(\text { direct sum) (see }[9 ; 1.17]) \text {. }
$$

In particular,

$$
G_{2}\left(Z_{\theta}\right)= \begin{cases}Z_{\theta}, & \text { if } \theta \text { is zero or odd } \\ Z_{2 \theta}, & \text { if } \theta \text { is a power of } 2 .\end{cases}
$$

We define a generator for $G_{2}\left(Z_{\theta}\right)$ by

$$
g_{2}\left(1_{\theta}\right)= \begin{cases}1_{\theta}, & \text { if } \theta \text { is zero or odd } \\ 1_{2 \theta}, & \text { if } \theta \text { is a power of } 2\end{cases}
$$

where $1_{r}=1 \bmod r(r=0,1, \cdots)$. The group $G_{2}\left(Z_{\theta}\right)$ will be the coefficient domain for the operation $\{$. As remarked in $[9 ; \S 2]$, once we have defined the operation $\mathfrak{p}$ for the category of regular cell complexes, the definition easily extends to the category of all topological spaces. Hence, in what follows we restrict attention to regular cell complexes, which we will simply term complexes.

Let $K$ be a complex and $L$ a subcomplex of $K$. Let $Z_{\theta}$ be a group in the category $\mathscr{C}$; that is, $\theta$ is zero or a power of a prime. We define an operation

$$
p: H^{q}\left(K, L ; Z_{\theta}\right) \longrightarrow H^{2 q+1}\left(K, L ; G_{2}\left(Z_{\theta}\right)\right)
$$

as follows. Let $u \in H^{q}\left(K, L ; Z_{\theta}\right)$; let $\beta$ be the homomorphism from $Z_{\theta}$ to $G_{2}\left(Z_{\theta}\right)$ given by $\beta\left(1_{\theta}\right)=\theta g_{2}\left(1_{\theta}\right)$. Define 


$$
p(u)=\beta_{*}\left(u \cup \delta_{*} u\right) .
$$

Here, $\delta_{*}$ is the Bockstein coboundary operator associated with the exact sequence

$$
0 \longrightarrow Z \stackrel{\theta}{\longrightarrow} Z \longrightarrow Z_{\theta} \longrightarrow 0,
$$

and the cup-product is taken relative to the natural pairing $Z_{\theta} \otimes Z \approx Z_{\theta}$.

It is easily seen that this agrees with the usual definition of the operation $p$ (see [3] and [10]). For let $\bar{u} \in C^{q}(K, L ; Z)$ be a cochain representing $u$; that is, $\delta \bar{u}=\theta \bar{v}$, for some cochain $\bar{v} \in C^{q+1}(K, L ; Z)$. Then, a cocycle representing $\beta_{*}\left(u \cup \delta_{*} u\right)$ is given by $\bar{u} \cup \delta \bar{u}$, which coincides with the definition given in [10].

In $[9 ; 8.14]$ we defined a function $w$ which goes from $H^{q}\left(K ; Z_{\theta}\right)$ to $H^{2 q+1}(K ; Z)$, This function can be extended to the relative case, following the method given in $\S 4$. When this is done it is easily shown that

$$
p(u)=\beta_{*} w(u),
$$

a result we will need later.

The Postnikov square, $\mathfrak{p}$, is defined using the operation $p$ as follows: let $u \in H^{q}\left(K, L ; A_{2 k}\right)$, where $\mathrm{A}_{2 k}$ is an even summand of a $p$-cyclic ring with divided powers. By hypothesis, $A_{2 k}$ is a cyclic group whose order is infinite or a power of a prime. Thus, there is an integer $\theta$ such that $A_{2 k}$ is isomorphic to $Z_{\theta}$, where $Z_{\partial} \in \mathcal{C}$. Let $\nu$ be an isomorphism from $A_{2 k}$ to $Z_{\theta}$. Then, by 3.1 in [9], for each non-negative integer $r$ we have defined a homomorphism $\zeta_{r}$ mapping $G_{r}\left(Z_{\theta}\right)$ to $A_{2 r k}$, which is an extension of $\nu^{-1}$. We define the operation $\mathfrak{p}$ by

$$
\mathfrak{p}(u)=\zeta_{2}^{*} p \nu_{*}(u) ;
$$

that is, $\mathfrak{p}$ is the composition of the following functions:

$$
\begin{gathered}
H^{q}\left(K, L ; A_{2 k}\right) \stackrel{\nu_{*}}{\longrightarrow} H^{q}\left(K, L ; Z_{\theta}\right) \stackrel{p}{\longrightarrow} \\
H^{2 q+1}\left(K, L ; G_{2}\left(Z_{\theta}\right)\right) \stackrel{\zeta_{2}^{*}}{\longrightarrow} H^{2 q+1}\left(K, L ; A_{4 k}\right) .
\end{gathered}
$$

We show the independence of this definition from the particular choice of the isomorphism $\nu$ (and hence $\zeta_{2}$ ). This is a consequence of the fact that

(2.4) Lemma.

$$
p \alpha_{*}=G_{2}(\alpha)_{*} p,
$$

where $\alpha$ is a homomorphism from $Z_{\theta}$ to a group $Z_{\tau}$ in 'b), and $G_{2}(\alpha)$ is the homomorphism from $G_{2}\left(Z_{9}\right)$ to $G_{2}\left(Z_{\tau}\right)$ induced by the functor $G$ (see $[9 ; 1.23])$.

Using 2.2, the proof of 2.4 is entirely similar to that given for 5.22 in [9] and is omitted here. From 2.4 the proof of the independence of 
the definition of $p$ follows along exactly the same lines as 3.5 and 3.6 in [9]; wo omit the details.

3. Suspension of cohomology operations. The definition of the suspension used here is due to N. E. Steenrod ${ }^{1}$. Let $I$ denote the unit interval, $[0,1]$, and $\dot{I}$ the subspace $\{0\} \cup\{1\}$. The group $H^{1}(I, \dot{I} ; Z)$ is cyclic infinite; let $v$ be a fixed generator. For each space $X$ and coefficient group $G$ define a function $\phi$ from $H^{q}(X ; G)$ to $H^{q+1}(I \times X, \dot{I} \times X ; G)$ by

$$
\phi(u)=v \times u .
$$

We use singular cohomology for $X$, and the natural pairing $Z \otimes G \approx G$ for the cross-product. In $\S 7$ we prove the following lemma.

(3.2) Lemma. The function $\phi$ is an isomorphism mapping $H^{q}(X ; G)$ onto $H^{q+1}(I \times X, \dot{I} \times X ; G)(q>0)$.

Consider now any cohomology operation $C$, which is defined on relative cohomology groups; say, $C$ maps $H^{r}(X, A ; G)$ to $H^{s}(X, A ; I I)$ for each pair $(X, A)$. Define an absolute cohomology operation, $S(C)$, which maps $H^{r-1}(Y ; G)$ to $H^{s-1}(Y ; I I)$, for each space $Y$, by

$$
S(C)(u)=\phi^{-1} C \phi(u) \quad\left(u \in H^{r-1}(Y ; G)\right) .
$$

Using the method described in $\S 4$ we may extend $S(C)$ to an operation defined on relative cohomology groups, an operation which we continue to denote by $S(C)$. We wish to apply this construction to the operation $\mathfrak{F}_{t}$; as defined in [9], this is just an absolute operation. Thus, to use Definition 3.3 we must first extend the definition of $i_{t}$ to the relative case.

4. Relative cohomology operations. Let $O(q, r ; G, I)$ denote the set of absolute cohomology operations relative to dimensions $q, r$ and coefficient groups $G, \Pi$; that is, if $C \in O(q, r ; G, \Pi)$, then $C: H^{q}(X ; G) \rightarrow$ $H^{r}(X, I)$ for each space $X$. As is well-known the set $O(q, r ; G, I I)$ is in 1-1 correspondance with the group $H^{r}(K ; I)$, where $K$ is an Eilenberg-MacLane space of type $(G, q)$. The correspondance is obtained by assigning $C(\iota)$ to $\iota$, where $\iota$ is the fundamental class in $H^{q}(K ; G)$. Choose now a base point $e \in K$, and let $\alpha^{*}: H^{*}(K, e ; \Lambda) \approx H^{*}(K ; A)$ be the isomorphism induced by the inclusion $K \subset(K, e)$. For any $C W$ complex $X$ and subcomplex $A$, the homotopy classes of maps $(X, A) \rightarrow(K, e)$

1 This definition has the advantage that it can be used in the case of cohomology with local coefficients. 
are in one-to-one correspondance with $H^{q}(X, A ; G)$. Thus we define a relative cohomology operation, $C^{\prime}$, associated with an absolute operation, $C$, as follows:

$$
C^{\prime}(u)=f^{*} \alpha^{*-1} C(\iota),
$$

where $u \in H^{u}(X, A ; G)$ and $f$ is a map $(X, A) \rightarrow(K, e)$ such that

$$
f^{*} \alpha^{*-1}(\iota)=u \text {. }
$$

With the operation $C^{\prime}$ defined, one is then interested in whether the properties of $C$ extend to the operation $C^{\prime}$. We now prove a general lemma which essentially asserts that all the properties of $C^{\prime}$ do carry over to $C^{\prime}$.

Let $O\left(q_{1}, \cdots, q_{n}, r ; G_{1}, \cdots, G_{n}, I l\right)$ denote the group of absolute cohomology operations, $T$, in $n$ variables; that is, if $u_{i} \in H^{q_{i}}\left(X ; G_{i}\right)$ $(i=1, \cdots, n)$, then, $T\left(u_{1}, \cdots, u_{n}\right) \in H^{r}(X ; I I)$. The operation $T$ extends to a relative operation, $T^{\prime}$, using the method just given for operations of a single variable. Suppose now we are given absolute cohomology operations

and

$$
\begin{aligned}
& C \in O\left(q_{1}, \cdots, q_{n}, r ; G_{1}, \cdots, G_{n}, \Pi\right), \\
& E \in O\left(s_{1}, \cdots, s_{n}, r ; I_{1}, \cdots, \Pi_{n}, \Pi\right), \\
& D_{i} \in O\left(q_{1}, \cdots, q_{n}, s_{i} ; G_{1}, \cdots, G_{n}, I I_{i}\right) \\
& \quad(i=1,2, \cdots, p) .
\end{aligned}
$$

Let $C^{\prime}, E^{\prime} D_{i}^{\prime}$, be the corresponding relative operations.

(4.2) Proposition. Suppose that for each space $X$ and cohomology classes $u_{i} \in H^{q_{i}}\left(X ; G_{i}\right)(i=1, \cdots, n)$, we have

$$
C\left(u_{1}, \cdots, u_{n}\right)=E\left(D_{1}\left(u_{1}, \cdots, u_{n}\right), \cdots, D_{p}\left(u_{1}, \cdots, u_{n}\right)\right) .
$$

Then, for each pair $(X, A)$ and classes $u_{i}^{\prime} \in H^{q_{i}}\left(X, A ; G_{i}\right)(i=1, \cdots, n)$, we have

$$
C^{\prime}\left(u_{1}^{\prime}, \cdots, u_{n}^{\prime}\right)=E^{\prime}\left(D_{1}^{\prime}\left(u_{1}^{\prime}, \cdots, u_{n}^{\prime}\right), \cdots, D_{p}^{\prime}\left(u_{1}^{\prime}, \cdots, u_{n}^{\prime}\right)\right) .
$$

We give the proof at the end of this section, first illustrating the theorem by giving several corollaries.

(4.3) Corollary 1. Let $C \in O(q, s ; R, S), D_{i} \in O\left(q_{i}, s_{i} ; R, S\right)$ $(i=1,2)$, where $R, S$ are rings, $q=q_{1}+q_{2}$, and $s=s_{1}+s_{2}$. Suppose that

$$
C\left(u_{1} \cup u_{2}\right)=D_{1}\left(u_{1}\right) \cup D_{2}\left(u_{2}\right)
$$

for all classes $u_{i} \in H^{q_{i}}(X ; R)$. Then, 


$$
C^{\prime}\left(u_{1}^{\prime} \cup u_{2}^{\prime}\right)=D_{1}^{\prime}\left(u_{1}^{\prime}\right) \cup D_{2}^{\prime}\left(u_{2}^{\prime}\right),
$$

for all classes $u_{i}^{\prime} \in\left(H^{q_{i}}(X, A ; R)\right.$.

Proof. Let $E_{R} \in O\left(q_{1}, q_{2}, q ; R, R, R\right)$ and $E_{S} \in O\left(s_{1}, s_{2}, s ; S, S, S\right)$ be the respective cup-products. Let $F$ be the composite operation $C \circ E_{R}$. Using Proposition 4.2 we see that $F^{\prime}=C^{\prime} \circ E_{k}^{\prime}$. But since $F\left(u_{1}, u_{2}\right)=$ $E_{S}\left(D_{1}\left(u_{1}\right), D_{2}\left(u_{2}\right)\right)$, again using 4.2 we see that

$$
F^{\prime}\left(u_{1}^{\prime}, u_{2}^{\prime}\right)=E_{S}^{\prime}\left(D_{1}^{\prime}\left(u_{1}\right), D_{2}\left(u_{2}^{\prime}\right)\right) ;
$$

that is,

$$
C^{\prime}\left(u_{1}^{\prime} \cup u_{2}^{\prime}\right)=D_{1}^{\prime}\left(u_{1}^{\prime}\right) \cup D_{2}^{\prime}\left(u_{2}^{\prime}\right),
$$

as was to be shown.

Let $C, D_{1}, D_{2}$ be the same operations as in Corollary 1. Then,

(4.4) Corollary 2. $C^{\prime}\left(u_{1}^{\prime} \times u_{2}^{\prime}\right)=D_{1}^{\prime}\left(u_{1}^{\prime}\right) \times D_{2}^{\prime}\left(u_{2}^{\prime}\right)$, where $u_{i} \in H^{q_{i}}\left(X_{i}, A_{i} ; R\right)$ $(i=1,2)$.

Proof. Let $p_{1}:\left(X_{1} \times X_{2}, A_{1} \times X_{2}\right) \rightarrow\left(X_{1}, A_{1}\right), p_{2}:\left(X_{1} \times X_{2}, X_{1} \times A_{2}\right) \rightarrow$ $\left(X_{2}, A_{2}\right)$ be projections. Then,

$$
u_{1}^{\prime} \times u_{2}^{\prime}=p_{1}^{*}\left(u_{1}^{\prime}\right) \cup p_{2}^{*}\left(u_{2}^{\prime}\right) .
$$

Thus,

$$
\begin{aligned}
C^{\prime}\left(u_{1}^{\prime} \times u_{2}^{\prime}\right) & =C^{\prime}\left(p_{1}^{*} u_{1}^{\prime} \cup p_{2}^{*} u_{2}^{\prime}\right)=D_{1}^{\prime}\left(p_{1}^{*} u_{1}^{\prime}\right) \cup D_{2}^{\prime}\left(p_{2}^{*} u_{2}^{\prime}\right) \\
& =p_{1}^{*}\left(D_{1}^{\prime} u_{1}^{\prime}\right) \cup p_{2}^{*}\left(D_{2}^{\prime} u_{2}^{\prime}\right)=\left(D_{1}^{\prime} u_{1}^{\prime}\right) \times\left(D_{2}^{\prime} u_{2}^{\prime}\right) .
\end{aligned}
$$

Here we have used Corollary 1 and the naturality of the cohomology operations involved.

To apply this to the operations $\mathfrak{x}_{\iota}$, recall the way in which these operations were defined (see $\S 3$ in [9]). We defined a set of "model operations", $P_{t}$, which used as coefficient groups only the groups of the category $\mathscr{C}$ (see $\S 2$ ). The operations $\mathfrak{F}_{t}$ were then defined by composing the operation $P_{t}$ with coefficient group homomorphisms; that is, precisely the same pattern as followed in Definition 2.3. Thus, the operations $\mathfrak{F}_{t}$, are defined in the relative case by simply applying the method given in this section to the operations $P_{t}$.

Let $P_{t}^{\prime}$ be the relative operation obtained from $P_{t}$. We note several facts needed later.

(4.5) Lemma. Let $u_{i} \in H^{q_{i}}\left(X_{i}, A_{i} ; Z_{\vartheta}\right)(i=1,2)$, where $Z_{\theta} \in \mathscr{C}$. Then

$$
P_{t}^{\prime}\left(u_{1} \times u_{2}\right)=P_{\iota}^{\prime}\left(u_{1}\right) \times P_{\iota}^{\prime}\left(u_{2}\right)(t \text { odd }) .
$$

If $t=2$ and $\theta$ is a power of 2 , then, 


$$
\begin{aligned}
& P_{2}^{\prime}\left(u_{1} \times u_{2}\right)=P_{2}^{\prime}\left(u_{1}\right) \times P_{2}^{\prime}\left(u_{2}\right) \\
& \quad+\nu_{*}\left[S q_{1}\left(u_{1}\right) \times \mu_{*} w\left(u_{2}\right)+\mu_{*} w\left(u_{1}\right) \times S q_{1}\left(u_{2}\right)\right] .
\end{aligned}
$$

Here, $\nu$ is the homomorphism of $Z_{2}$ to $G_{2}\left(Z_{\theta}\right)$ given by $\nu\left(1_{2}\right)=\theta g_{2}\left(1_{\theta}\right)$, and $\mu$ is the factor homomorphism $Z_{0} \rightarrow Z_{2}$. The functions $S q$ and $w$ are defined respectively in 9.6 and 8.14 of [9].

Proof. The first statement is a consequence of Corollary 4.3 and the fact that the absolute operations $P_{t}$ satisfy this formula ${ }^{2}$. Equation 4.5(2) was remarked in $[9 ; \S 13]$ for the absolute operations $P_{t}$, and the case $\operatorname{dim} u_{i}$ odd. But it follows from 8.12 in [9] that 4.5(2) holds in general. In fact Theorem 8.11 in [9] can be obtained at once from equation 4.5(2). The extension of the equation to the relative operation $P_{t}^{\prime}$, follows then from application of Proposition 4.2.

Combining Proposition 4.2 and 8.2 of [9] we also obtain

(4.6) Lemma. Let $t$ be an an integer where $t=p_{h} \cdots p_{1}\left(p_{i}\right.$ prime). Let $u \in H^{2 q}(X, A ; Z)$. $(Z, \in \mathscr{C})$. Then,

$$
P_{\mathrm{t}}^{\prime}(u)=P_{p_{k}}^{\prime} \circ \cdots \circ P_{p_{1}}^{\prime}(u) .
$$

Since it is in fact the relative operation, $P_{t}^{\prime}$, we will work with, from now on we drop the prime, writing only $P_{t}$ for both the relative and absolute operation.

Proof of Proposition 4.2. Let $Y=K\left(G_{1}, q_{1}\right) \times \cdots \times K\left(G_{n}, q_{n}\right)$, where each $K\left(G_{i}, q_{i}\right)$ is on Eilenberg-MacLane space of type $\left(G_{i}, q_{i}\right)$. Let $\pi_{j}: Y \rightarrow K\left(G_{j}, q_{j}\right)(j=1, \cdots, n)$, be the projection map and set $\bar{\iota}_{j}=\pi_{j}^{*}\left(\iota_{j}\right)$, where $\iota_{j}$ is the characteristic class in $H^{q_{j}},\left(K\left(G_{j}, q_{j}\right) ; G_{j}\right)$. Let $e_{j}$ be a base point in $K\left(G_{j}, q_{j}\right)$ and set $Y^{\prime}=\left(K\left(G_{1}, q_{1}\right), e_{1}\right) \times \cdots \times$ $\left(K\left(G_{n}, q_{n}\right), e_{n}\right)$. Let $\iota_{j}^{\prime}, \bar{\iota}_{j}^{\prime}$ be the equivalent of $\iota_{j}$ and $\bar{\iota}_{j}$. Then, Proposition 4.2 follows at once from the following three lemmas (we keep the same notation as used in Proposition 4.2)

$$
C\left(u_{1}, \cdots, u_{n}\right)=E\left(D_{1}\left(u_{1}, \cdots, u_{n}\right), \cdots, D_{p}\left(u_{1}, \cdots, u_{n}\right)\right)
$$

if and only if

$$
C\left(\bar{\iota}_{1}, \cdots, \bar{\iota}_{n}\right)=E\left(D_{1}\left(\bar{\iota}_{1}, \cdots, \bar{\iota}_{n}\right), \cdots, D_{p}\left(\bar{\iota}_{1}, \cdots, \bar{\iota}_{n}\right)\right) .
$$

if and only if

$$
C^{\prime}\left(\bar{\iota}_{1}^{\prime}, \cdots, \bar{\iota}_{n}^{\prime}\right)=E^{\prime}\left(D_{1}^{\prime}\left(\bar{\iota}_{1}, \cdots, \bar{\iota}_{n}^{\prime}\right), \cdots, D_{p}^{\prime}\left(\bar{\iota}_{1}^{\prime}, \cdots, \bar{\iota}_{n}^{\prime}\right)\right)
$$

${ }^{2}$ The operations $\mathfrak{P}_{\ell}$ are easily defined for odd dimensional classes: see $[\mathbf{9} ; \$ 7]$. 


$$
\text { If } C\left(\bar{\iota}_{1}, \cdots, \bar{\iota}_{n}\right)=E\left(D_{1}\left(\bar{\iota}, \cdots, \bar{\iota}_{n}\right), \cdots, D_{p}\left(\bar{\iota}_{1}, \cdots, \bar{\iota}_{n}\right)\right)
$$

then,

$$
C^{\prime}\left(\bar{\iota}_{1}^{\prime}, \cdots, \bar{\iota}_{n}^{\prime}\right)=E^{\prime}\left(D_{1}^{\prime}\left(\bar{\iota}_{1}^{\prime}, \cdots, \bar{\iota}_{n}^{\prime}\right), \cdots, D_{p}^{\prime}\left(\bar{\iota}_{1}^{\prime}, \cdots, \bar{\iota}_{n}^{\prime}\right)\right) .
$$

We give only the proof of Lemma 4.7, the others being entirely similar. Assume first we are given classes $u_{i} \in H^{q_{i}}\left(X ; G_{i}\right)(i=1, \cdots, n)$. Let $f_{j}: X \rightarrow K\left(G_{j} ; q_{j}\right)$ be mappings such that $f_{j}^{*}\left(c_{j}\right)=u_{j}$. Set $f=f_{1} \times$ $\cdots \times f_{n}: X \rightarrow Y$. Then, by naturality, one has

$$
\text { (a) } \quad C\left(u_{1}, \cdots, u_{n}\right)=f^{*} C\left(\bar{\iota}_{1}, \cdots, \bar{\iota}_{n}\right) \text {, }
$$

$$
D_{\imath}\left(u_{1}, \cdots, u_{n}\right)=f^{*} D_{i}\left(\bar{\iota}_{1}, \cdots, \bar{\iota}_{n}\right)
$$$$
(i=1, \cdots, p) \text {. }
$$

Suppose now that

$$
C\left(\bar{\iota}_{1}, \cdots, \bar{\iota}_{n}\right)=E\left(D_{1}\left(\bar{\iota}_{1}, \cdots, \bar{\iota}_{n}\right), \cdots, D_{p}\left(\bar{\iota}_{1}, \cdots, \bar{\iota}_{n}\right)\right) .
$$

Then, by 4.10 ,

$$
C\left(u_{1}, \cdots, u_{n}\right)=f^{*} E\left(D_{1}\left(\bar{\iota}_{1}, \cdots, \bar{\iota}_{n}\right), \cdots, D_{p}\left(\bar{\iota}_{1}, \cdots, \bar{\iota}_{n}\right)\right) .
$$

But $E$ is natural with respect to mappings. Therefore,

$$
\begin{aligned}
& f^{*} E\left(D_{1}\left(\bar{\iota}_{1}, \cdots, \bar{\iota}_{n}\right), \cdots, D_{p}\left(\bar{\iota}_{1}, \cdots, \bar{\iota}_{n}\right)\right) \\
= & E\left(f^{*} D_{1}\left(\bar{\iota}_{1}, \cdots, \bar{\iota}_{n}\right), \cdots, f^{*} D_{p}\left(\bar{\iota}_{1}, \cdots, \bar{\iota}_{n}\right)\right) \\
= & E\left(D_{1}\left(u_{1}, \cdots, u_{n}\right), \cdots, D_{p}\left(u_{1}, \cdots, u_{n}\right)\right),
\end{aligned}
$$

again by 4.10 , which completes the proof of this assertion. The proof in the opposite direction is trivial.

5. The proof of Theorem I. Recall that the operation $\mathfrak{H}_{t}$ is defined by means of the model operations $P_{t}$ and coefficient group homomorphisms. But it is clear that the isomorphism $\phi$, defined in 3.1 , commutes with coefficient group homomorphisms. Thus, it suffices to prove Theorem I with $\mathfrak{B}_{t}$ replaced by $P_{t}$, the operation $\mathfrak{i}$ replaced by $p$, and the group $A_{2 k}$ taken to be a group in the category $\mathscr{C}$, say $A_{2 k}=Z_{\theta}$.

Assume first that $t$ is an odd prime $p$. Since $\phi$ is an isomorphism, the proof of Theorem I (ii) consists simply in showing

$$
P_{p} \phi(u)=0, \quad u \in H^{r}\left(X ; Z_{\theta}\right) .
$$

But this is immediate; for

$$
P_{p} \phi(u)=P_{p}(v \times u)=P_{p}(\bar{v} \times u)=P_{p}(\bar{v}) \times P_{p}(u),
$$

by Lemma $4.5(1)$. Here, $\bar{v}$ is a generator of $H^{1}\left(I, \dot{I} ; Z_{\vartheta}\right)$. However, $P_{p}(\bar{v})=0$, by dimensionality considerations. Thus, $P_{p} \phi(u)=0$; and hence, $S\left(P_{p}\right)=0$. 
Now, suppose that $t$ is any integer $>1$ which is not a power of 2; say, $t=m p$, where $p$ is an odd prime. Then, by Lemma 4.6

$$
P_{t} \phi(u)=P_{m} \circ P_{p} \phi(u)=P_{m}(0)=0 .
$$

Consequently,

$$
S\left(P_{t}\right)=0 \text {. }
$$

Thus, we have proved Theorem $I$ (ii) for the case $t$ is not a power of 2. Before concluding the proof of part (ii), we must prove part (i). Let the classes $u$ and $v$ be as above, where $u$ has coefficients in the group $Z_{\theta}$. If $\theta$ is zero or odd, then by Proposition 7.4 in [9], we have

$$
P_{2}(v \times u)=P_{2}(\bar{v} \times u)=(\bar{v} \times u)^{2}= \pm \bar{v}^{2} \times u^{2}=0,
$$

since $\bar{v}^{2}=0$. Thus, in this case $S\left(P_{2}\right)=0$. Suppose now that $\theta$ is a power of 2 .

Let $\eta$ be the factor map $Z \rightarrow Z_{\theta}$. Then, $v \times u=\left(\eta_{*} v\right) \times u$, where the right hand side uses the pairing $Z_{\theta} \otimes Z_{\theta} \approx Z_{\theta}$. Thus, using Lemma $4.5(2)$, we have

$$
\begin{aligned}
& P_{2}(v \times u)=P_{2}\left(\eta_{*} v \times u\right)=P_{2}\left(\eta_{*} v\right) \times P_{2}(u) \\
& \quad+\nu_{*}\left[S q_{1}\left(\eta_{*} v\right) \times \mu_{*} w(u)+\mu_{*} w\left(\eta_{*} v\right) \times S q_{1}(u)\right] .
\end{aligned}
$$

Now, $P_{2}\left(\eta_{*} v\right)=0, w\left(\eta_{*} v\right)=0$ by dimensionality considerations. Also, since $\eta_{*} v$ is a 1 -dimensional class, $S q_{1}\left(\eta_{*} v\right)=\xi_{*} v$, where $\xi$ is the natural $\operatorname{map} Z \rightarrow Z_{2}$ (see Steenrod [4; 12.6]). Thus,

$$
P_{2}(v \times u)=\nu_{*}\left[\xi_{*} v \times \mu_{*} w(u)\right] .
$$

Consider the following commutative diagram:

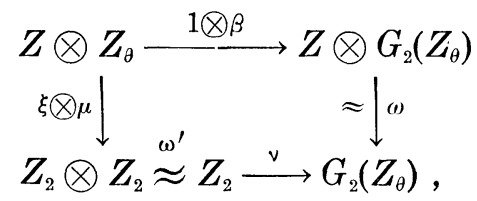

where $\beta$ is the homomorphism of $Z_{\theta}$ to $G_{2}\left(Z_{\theta}\right)$ given by $\beta\left(1_{\theta}\right)=\theta g_{2}\left(1_{\theta}\right)$ (see 2.1). Then, from 5.1,

$$
\begin{aligned}
P_{2}(v \times u) & =\nu_{*}\left(\omega_{*}^{\prime}(\zeta \otimes \mu)_{*}[v \otimes w(u)]\right. \\
& =\omega_{*}(1 \otimes \beta)_{*}[v \otimes w(u)] \\
& =v \times \beta_{*} w(u) \\
& =v \times p(u), \text { by } 2.2 .
\end{aligned}
$$

Therefore, 


$$
P_{2} \phi(u)=P_{2}(v \times u)=v \times p(u)=\phi p(u) .
$$

That is,

$$
S\left(P_{2}\right)=p .
$$

This proves part (i) of Theorem I. To complete the proof of the theorem we must show that

$$
P_{2^{r}} \phi(u)=0, \quad(r>1) .
$$

But by part (i) of Theorem I and Lemma 4.6, we have

$$
\begin{aligned}
P_{2^{r}} \phi(u) & =P_{2^{r-1}} P_{2} \phi(u)=P_{2^{r-1}} \phi p(u) \\
& =P_{2^{r-2}} P_{2} \phi p(u)=P_{2^{r-2}} \phi p(p(u))=0 .
\end{aligned}
$$

Here, we use property 6.6 of the function $p$, which is proved independently in the next section. This completes the proof of Theorem I.

6. The properties of the operation $\mathfrak{k}$. We give here the main properties of the Postnikov square, $p$.

(6.1) Theorem. Let $X$ be a space, and let $A=\sum_{k} A_{k}$ be a p-cyclic ring with divided powers. Suppose that $u \in H^{q}\left(X ; A_{2 k}\right)(q, k>0)$. Then, ${ }^{3}$

$$
\begin{gathered}
\mathfrak{p}(u)=0, \text { if order } A_{2 k} \text { is odd or infinite, } \\
2 \mathfrak{p}(u)=0
\end{gathered}
$$

$\mathfrak{p}$ is a homomorphism,

$$
\begin{gathered}
\text { if order } A_{2 k}=2^{i}(i>1) \text { and } 2 u=0, \text { then } \mathfrak{p}(u)=0, \\
\mathfrak{p}(\mathfrak{p}(u))=0, \\
f^{*} \mathfrak{p}(u)=\mathfrak{p f} f^{*}(u), \\
\alpha_{* \mathfrak{p}}(u)=\mathfrak{p} \alpha_{*}(u),
\end{gathered}
$$

where $f^{*}$ is induced by a map $f$ from a space $Y$ to $X$, and $\alpha_{*}$ is induced by a homomorphism $\alpha$ from $A$ to a p-cyclic ring with divided powers $A^{\prime}$.

The proof of Theorem 6.1 falls into 2 parts. Suppose first that we have proved 6.2 through 6.7 with the operation p replaced by the operation $p$, and the coefficient group $A_{2 k}$ restricted to be a group in the category $\mathscr{C}$. Then, the proof of $6.2-6.7$ for the general case of the

$3 \mathrm{~W}$ ith the exception of 6.5 and 6.6 , these properties are noted by J. H. C. Whitehead in $\lceil\mathbf{1 0}\rceil$. 
function $\mathfrak{p}$ follows at once, using definition 2.3 ; that is, $\mathfrak{p}=\zeta_{2} * p \nu_{*}$. In particular, $6.2-6.5$ are simple consequences of the fact that $\zeta_{2} *$ and $\nu_{*}$ are homomorphisms; 6.6 follows from 6.3 and 6.5 , and 6.7 follows from the fact that $f^{*}$ commutes with all coefficient group homomorphisms. Finally, to prove 6.8 for the operation $\mathfrak{p}$, one uses 2.4 and exactly the same argument as that used to prove $\mathrm{I}(9)$ in $\S 4$ of [9]. Thus, we are left with proving 6.2 through 6.7 for the operation $p$. Let $u \in H^{4}(K ; Z)$, where $Z_{\theta} \in \mathscr{C}$. Then,

$$
p(u)=0 \text {, if } \theta \text { is zero or odd. }
$$

This follows at once from 2.1. For if $\theta$ is zero or odd, the homomorphism $\beta$ is zero.

$$
2 p(u)=0
$$

This again is immediate from 2.1; for it is always the case that $2 \beta=0$.

$$
p \text { is a homomorphism }
$$

In $\S 5$ we showed that the operation $p$ is the suspension of the operation $P_{2}$. But by 7.4 in [6], all operations which are suspensions are homomorphisms.

$$
\text { If } \theta=2^{i}(i>1) \text {, and } 2 u=0 \text {, then } p(u)=0 .
$$

Since $2 u=0$, we may use Lemma 13.3 of [9]: namely, there are classes $x \in H^{q-1}\left(K ; Z_{2}\right)$ and $y \in H^{q}\left(K ; Z_{2}\right)$ such that

$$
u=\lambda_{*} \delta_{*}(x)+\nu_{*}(y),
$$

where $\delta_{*}$ is the coboundary associated with the exact sequence

$$
0 \longrightarrow Z \stackrel{2}{\longrightarrow} Z \longrightarrow Z_{2} \longrightarrow 0,
$$

$\lambda$ is the natural factor map $Z \rightarrow Z_{3}$, and $\nu$ maps $Z_{2}$ to $Z_{\theta}$ by $\nu\left(1_{2}\right)=$ $(\theta / 2) 1_{\theta}$ (recall that $\theta=2^{i}, i>1$ ). Hence, by (iii) above,

$$
\begin{aligned}
p(u) & =p \lambda_{*} \delta_{*}(x)+p \nu_{*}(y) \\
& =G_{2}(\lambda)_{*} p \delta_{*}(x)+G_{2}(\nu)_{*} p(y) \\
& =G_{2}(\nu)_{*} p(y),
\end{aligned}
$$

by 2.4 and (i) above, since $\delta_{*}(u)$ has integer coefficients. Now,

$$
G_{2}(\nu)_{*} p(y)=G_{2}(\nu)_{*} \beta_{*} w(u),
$$

by 2.2. We show that $p(u)=0$ by showing that

$$
G_{2}(\nu) \beta=0 .
$$


From Definition 2.1 we recall that $\beta$ maps $Z_{2}$ to $G_{2}\left(Z_{2}\right)$ by $\beta\left(1_{2}\right)=$ $2 g_{2}\left(1_{2}\right)$. Hence, using 1.21 and 1.24 in [9],

$$
\begin{aligned}
G_{2}(\nu) \beta\left(1_{2}\right) & =2 G_{2}(\nu) g_{2}\left(1_{2}\right)=2 g_{2}\left(\nu 1_{2}\right) \\
& =2 g_{2}\left((\theta / 2) 1_{\theta}\right)=2\left(\theta^{2} / 4\right) g_{2}(1)=\left(\theta^{2} / 2\right) 1_{2 \theta}=0 .
\end{aligned}
$$

For, $\theta^{2} / 2=2^{2 i} / 2=2^{2 i-1}$; and, $2 \theta=2^{i+1}$. But by hypothesis, $i \geqq 2$; thus $2 i-1 \geqq i+1$.

$$
p(p(u))=0
$$

This follows at once from (ii) and (iv) above.

$$
f^{*} p(u)=p f^{*}(u) \text {. }
$$

This is simply a special case of Theorem 3.6 of [7]. This, then completes the proof of Theorem 6.1.

We consider one more property of the operation $p$ : namely, its behaviour with respect to suspension. We continue to denote by $S(C)$ the suspension of a cohomology operation $C$.

(6.9) Proposition. $S(\mathfrak{p})=0$, where 0 denotes the trivial cohomology operation.

Proof. By the same reasoning as given in $\S 5$, it sufficies to prove Proposition 6.9 with $p$ replaced by the operation $p$, and the coefficient group $A_{2 k}$ taken to be a group in the category say $A_{2 k}=Z$. Thus, we need simply show that $p \phi(u)=0$, where $u \in H^{q}\left(L ; Z_{3}\right)$. Now by Nakaoka [2] we have":

$$
p\left(v_{1} \times v_{2}\right)=P_{2}\left(v_{1}\right) \times p\left(v_{2}\right)+p\left(v_{1}\right) \times P_{2}\left(v_{2}\right),
$$

for classes $v_{i} \in H^{q_{i}}\left(X_{i}, A_{i} ; Z\right)(i=1,2)$.

Thus,

$$
p \phi(u)=p(\bar{v} \times u)=P_{2}(\bar{v}) \times p(u)+p(\bar{v}) \times P_{2}(u)=0,
$$

since $P_{2}(\bar{v})=p(\bar{v})=0$ by dimensionality considerations. Here, $\bar{v}$ is the image of $v$ in $H^{1}\left(I, \dot{I} ; Z_{\theta}\right)$. Hence, $S(p)=0$, as was to be proved.

7. The relation $\delta S(C)=C \delta$. We give here a theorem, whose proof is due to N. E. Steenrod.

(7.1) Theorem. Let $C$ be a cohomology operation, and let $\delta$ be the relative cohomology coboundary operator. Then,

${ }^{4}$ Nakaoka only proves this for the case $\operatorname{dim} v_{1}, v_{2}$ even; but the result is true in general, as is easily shown using Definition 2.1. 


$$
\delta S(C)=C \delta,
$$

where $S(C)$ is the suspension of $C$.

We sketch the proof; let $X$ be a space and $A \subset X$ a subspace. Let $X^{\prime}$ denote the mapping cylinder of the inclusion map $A \subset X$. That is, unite $I \times A$ and $X$ by identifying $1 \times A$ with $A$ in $X$. Let $A^{\prime}=0 \times A$. The inclusions

$$
\left(X^{\prime}, A^{\prime}\right) \longrightarrow\left(X^{\prime}, I \times A\right) \longleftarrow(X, A)
$$

induce isomorphisms of the cohomology sequence of $(X, A)$ and $\left(X^{\prime}, A^{\prime}\right)$ with local cosfficients. Thus, we may discuss the behaviour of the coboundary $\delta$ in the cohomology sequence of the pair $\left(X^{\prime}, A^{\prime}\right)$.

Consider the following hexagonal diagram (see [8], page 42 ):

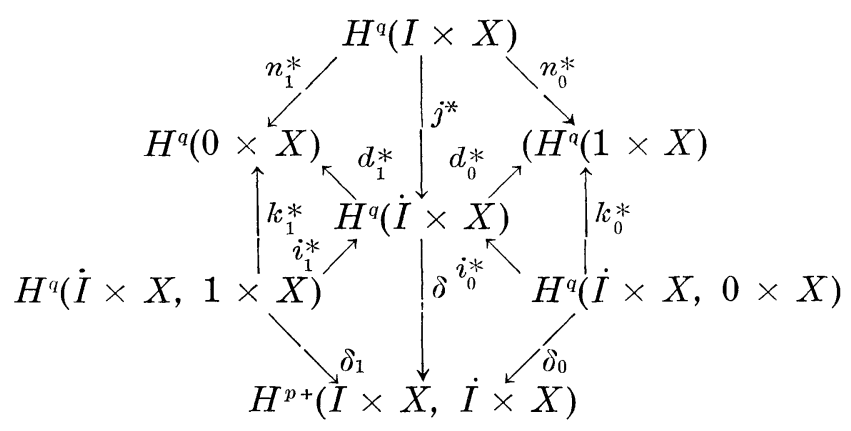

Here all homomorphisms other than $\delta, \delta_{1}$, and $\delta_{2}$ are induced by inclusions. Standard arguments, using exactness and homotopy equivalence, show that the arrows around the peripheries are isomorphisms. We agree to identify $H^{q}(X)$ with $H^{q}(0 \times X)$ by sending $u \rightarrow e \times u$, where $e$ is the unit of $H^{\circ}(0 ; Z)$. At the end of this section we will use diagram 7.2 to prove the following lemma:

(7.3) Lemma. Let $\phi$ be the function defined in 3.1. Then,

$$
\phi=\delta_{1} k_{1}^{*-1},
$$

where $k_{1}^{*}, \delta_{1}$ are the functions defined in diagram 7.2

Notice that this proves Lemma 3.2; for the functions $\delta_{1}, k_{1}^{*}$ are isomorphisms. Now let $g^{*}: H^{q+1}\left(X^{\prime}, A^{\prime} \cup X\right) \rightarrow H^{q+1}(I \times A, \dot{I} \times A)$ be induced by the inclusion. Using the fact that $\dot{I}$ is a strong deformation retract of a neighborhood of $\dot{I}$ in $I$ (see [8]; Chapter 1, 11.6), together with excision, one shows that $g^{*}$ is an isomorhism onto.

(7.4) Lemma. The following diagram is commutative, where $f^{*}$ is induced by the inclusion 


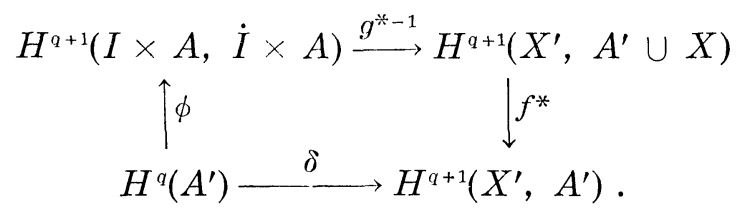

Thus $\delta=f^{*} g^{*-1} \phi$.

This is a consequence of Lemma 7.3 and commutativity relations in a slightly enlarged diagram. We omit the details.

The proof of Theorem 7.1 is an immediate consequence of Lemma 7.4. For let $u \in H^{q}\left(A^{\prime}\right)$. Then, by this lemma,

$$
C \delta(u)=C f^{*} g^{*-1} \phi(u) .
$$

Using the naturality of the operation $C$, we have

$$
C f^{*} g^{*-1} \phi(u)=f^{*} g^{*-1} C \phi(u) .
$$

But by Definition 3.1, $C \phi=\phi S(C)$.

Thus,

$$
C \delta(u)=f^{*} g^{*-1} \phi S(C)(u)=\delta S(C)(u),
$$

again using Lemma 7.4. This completes the proof of Theorem 7.1.

Proof of Lemma 7.3. We apply diagram 7.2 to the case $X=\varnothing$, $q=0$, and coefficient group $=$ integers. Then, the unit class of $H^{\circ}(\dot{I} ; Z)$ can be represented as a sum $v_{0}+v_{1}$, where

$$
v_{0}=i_{1}^{*} k_{1}^{*-1} d_{1}^{*}\left(v_{0}+v_{1}\right), v_{1}=i_{0}^{*} k_{0}^{*-1} d_{0}^{*}\left(v_{0}+v_{1}\right) .
$$

Thus,

$$
\delta\left(v_{0}\right)=-\delta\left(v_{1}\right)=v=\text { a generator of } H^{1}(I, \quad \dot{I} ; Z) \text {. Therefore, by }
$$
Definition 3.1,

$$
\phi(u)=v \times u=\left(\delta v_{0}\right) \times u .
$$

But by the axioms for the cross-product, we may write

$$
\left(\delta v_{0}\right) \times u=\delta\left(v_{0} \times u\right) .
$$

Furthermore, we have

$$
v_{0}=i_{1}^{*} k_{1}^{*-1}(e),
$$

where $e=d_{1}^{*}\left(v_{0}+v_{1}\right)=$ unit of $H^{0}(0 ; Z)$. Thus,

$$
\begin{aligned}
\delta\left(v_{0} \times u\right) & =\delta\left(i_{1}^{*} k_{1}^{*-1}(e) \times u\right) \\
= & \delta i_{1}^{*} k_{1}^{*-1}(e \times u)=\delta_{1} k_{1}^{*-1}(e \times u) .
\end{aligned}
$$


Here we have used the naturality of the cross-product and the commutativity of diagram 7.2. If we now identify $H^{q}(X)$ with $H^{q}(0 \times X)$ by sending $u \rightarrow e \times u$, we then have

$$
\phi(u)=\delta\left(v_{0} \times u\right)=\delta_{1} k_{1}^{*-1}(u),
$$

as was asserted.

\section{REFERENCES}

1. H. Cartan, Seminaire H. Cartan 1954/55, Paris (mimeographed).

2. M. Nakaoka, Note on cohomological operaíions, J. Inst. Polytech., Osaka City Univ., 4 (1953), 51-58.

3. M. Postnikov, The classification of continuous mappings of a 3-dimensional polyhedron iuio a simply connected polyhedron of arbitrary dimension, C. R. (Doklady) Acad. Sci. U. R. S. S., 64(1949), 461-462.

4. N. Steenrod, Products of cocycles and extensions of mappings, Ann. Math., 48 (1947), 290-320.

5. Homology groups of symmetric groups and reduced operations, Proc. Nat. Acad. Sci. U. S. A., 39 (1953), 213-223.

6 . Cohomology operations, Symposium Int. Topologie algebraica, Univ. de Mexico (1957), (to appear).

7. - Cohomology operations derived from the symmetric group, Comment. Math. Helv., 31 (1957), 195-218.

8. N. Steenrod and E. Eilenberg, Foundations of Algobraic Topology, Princeton University Press, 1952.

9. E. Thomas, The generalized Pontrjagin cohomology operations and rings with divided powers, Memoir Number 27 A. M. S. (1957).

10. J. H. C. Whitehead, On the theory of obstructions, Ann. Math., 54 (1951), 68-84.

UNIVERSITY OF CALIFORNIA

Berkeley, California 



\section{PACIFIC JOURNAL OF MATHEMATICS}

\section{EDITORS}

\section{David Gilbarg}

Stanford University Stanford, California

\section{R. A. Beaumont}

University of Washington

Seattle 5, Washington

\section{A. L. Whiteman}

University of Southern California Los Angeles 7, California

L. J. Paige

University of California

Los Angeles 24, California

\section{ASSOCIATE EDITORS}

E. F. BECKENBACH
C. E. BURGESS
E. HEWITT
A. HORN

A. HORN

\author{
V. GANAPATHY IYER \\ R. D. JAMES \\ M. S. KNEBELMAN \\ L. NACHBIN
}

I. NIVEN

T. G. OSTROM

H. L. ROYDEN

M. M. SCHIFFER
E. G. STRAUS

G. SZEKERES

F. WOLF

K. YOSIDA

\section{SUPPORTING INSTITUTIONS}

\author{
UNIVERSITY OF BRITISH COLUMBIA \\ CALIFORNIA INSTITUTE OF TECHNOLOGY \\ UNIVERSITY OF CALIFORNIA \\ MONTANA STATE UNIVERSITY \\ UNIVERSITY OF NEVADA \\ OREGON STATE COLLEGE \\ UNIVERSITY OF OREGON \\ OSAKA UNIVERSITY \\ UNIVERSITY OF SOUTHERN CALIFORNIA
}

\author{
STANFORD UNIVERSITY \\ UNIVERSITY OF TOKYO \\ UNIVERSITY OF UTAH \\ WASHINGTON STATE COLLEGE \\ UNIVERSITY OF WASHINGTON \\ * * * * \\ AMERICAN MATHEMATICAL SOCIETY \\ CALIFORNIA RESEARCH CORPORATION \\ HUGHES AIRCRAFT COMPANY \\ SPACE TECHNOLOGY LABORATORIES
}

Mathematical papers intended for publication in the Pacific Journal of Mathematics should be typewritten (double spaced), and the author should keep a complete copy. Manuscripts may be sent to any one of the four editors. All other communications to the editors should be addressed to the managing editor, L. J. Paige at the University of California, Los Angeles 24, California.

50 reprints per author of each article are furnished free of charge; additional copies may be obtained at cost in multiples of 50 .

The Pacific Journal of Mathematics is published quarterly, in March, June, September, and December. The price per volume (4 numbers) is $\$ 12.00$; single issues, $\$ 3.50$. Back numbers are available. Special price to individual faculty members of supporting institutions and to individual members of the American Mathematical Society: $\$ 4.00$ per volume; single issues, $\$ 1.25$.

Subscriptions, orders for back numbers, and changes of address should be sent to Pacific Journal of Mathematics, 2120 Oxford Street, Berkeley 4, California.

Printed at Kokusai Bunken Insatsusha (International Academic Printing Co., Ltd.), No. 6, 2-chome, Fujimi-cho, Chiyoda-ku, Tokyo, Japan.

PUBLISHED BY PACIFIC IOURNAL OF MATHEMATICS, A NON-PROFIT CORPORATION

The Supporting Institutions listed above contribute to the cost of publication of this Journal, but they are not owners or publishers and have no responsibility for its content or policies. 


\section{Pacific Journal of Mathematics}

\section{Vol. 9, No. $3 \quad$ July, 1959}

Errett Albert Bishop, A minimal boundary for function algebras . . . . . . . . . . . . 629

John W. Brace, The topology of almost uniform convergence . . . . . . . . . . . . 643

Cecil Edmund Burgess, Chainable continua and indecomposability .......... 653

L. Carlitz, Multiplication formulas for products of Bernoulli and Euler

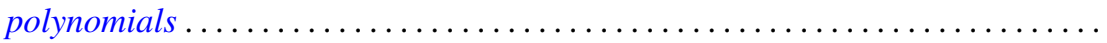

Eckford Cohen, A class of residue systems (mod $r$ ) and related arithmetical

functions. II. Higher dimensional analogues ....................

Shaul Foguel, Boolean algebras of projections of finite multiplicity . . . . . . . . . .

Richard Robinson Goldberg, Averages of Fourier coefficients .................

Seymour Goldberg, Ranges and inverses of perturbed linear operators .

Philip Hartman, On functions representable as a difference of convex functions ....

Milton Vernon Johns, Jr. and Ronald Pyke, On conditional expectation and

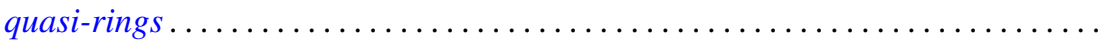

Robert Jacob Koch, Arcs in partially ordered spaces ....................

Gregers Louis Krabbe, A space of multipliers of type $L^{p}(-\infty, \infty) \ldots \ldots \ldots \ldots$

John W. Lamperti and Patrick Colonel Suppes, Chains of infinite order and their

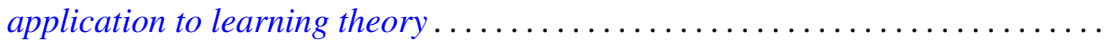

Edith Hirsch Luchins, On radicals and continuity of homomorphisms into Banach

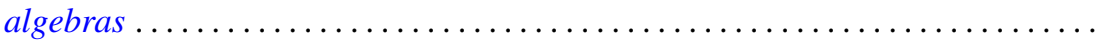

T. M. MacRobert, Multiplication formulae for the E-functions functions of their parameters.

Michael Bahir Maschler, Classes of minimal and representative domains and their kernel functions.

William Schumacher Massey, On the imbeddability of the real projective spaces in Euclidean space.

Thomas Wilson Mullikin, Semi-groups of class $\left(C_{0}\right)$ in $L_{p}$ determined by parabolic differential equations

Steven Orey, Recurrent Markov chains

Ernest Tilden Parker, On quadruply transitive groups ........ . .

Calvin R. Putnam, On Toeplitz matrices, absolute continuity, and unitary

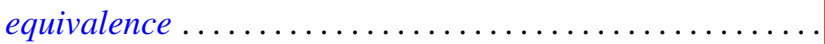

Helmut Heinrich Schaefer, On nonlinear positive operators.

Robert Seall and Marion Wetzel, Some connections between continued fractions and convex sets

Robert Steinberg, Variations on a theme of Chevalley

Olga Taussky and Hans Zassenhaus, On the similarity transformation between a



Emery Thomas, The suspension of the generalized Pontrjagin cohomology



Joseph L. Ullman, On Tchebycheff polynomials ..................... 913

Richard Steven Varga, Orderings of the successive overrelaxation scheme ........ 925

Orlando Eugenio Villamayor, Sr., On weak dimension of algebras . 